REGARDS

SUR L'ECONOMIE ALLEMANDE

BULLETIN ECONOMIQUE DU CIRAC
Regards sur l'économie allemande

Bulletin économique du CIRAC

$71 \mid 2005$

Varia

\title{
Aufbau Ost : entre désillusion, dilemme et renouveau
}

Markus Gabel

\section{OpenEdition}

\section{Journals}

Édition électronique

URL : http://journals.openedition.org/rea/327

DOI : $10.4000 /$ rea. 327

ISBN : 978-2-8218-0838-6

ISSN : 1965-0787

Éditeur

CIRAC

\section{Édition imprimée}

Date de publication : 1 mai 2005

Pagination : 15-24

ISSN : 1156-8992

\section{Référence électronique}

Markus Gabel, «Aufbau Ost : entre désillusion, dilemme et renouveau », Regards sur l'économie allemande [En ligne], 71 | mai 2005, mis en ligne le 24 juillet 2015, consulté le 24 avril 2019. URL http://journals.openedition.org/rea/327 ; DOI : 10.4000/rea.327 


\section{Aufbau Ost : entre désillusion, dilemme et renouveau}

Markus Gabel

Confrontée à la difficulté de comprimer son déficit d'origine structurelle, l'Allemagne débat vivement de la situation économique et sociale des nouveaux Länder. Le volume et l'orientation de la politique de reconstruction lancée après l'unification (Aufbau Ost) ne sont plus des sujets tabous face à un triple constat. D'un côté, cette politique a modernisé les régions de l'ex-RDA : le niveau de vie de la population s'est nettement élevé, et des infrastructures d'excellente qualité ont été créées. Ces succès sont le résultat d'investissements publics très élevés, d'un soutien massif aux entreprises, d'un cadre institutionnel de bonne qualité et d'importants transferts sociaux. D'un autre côté, malgré ces efforts, le processus de rattrapage s'est arrêté depuis maintenant plus de 8 ans. Dans certains domaines, il pourrait même s'inverser à l'avenir, car les perspectives de croissance de l'est paraissent limitées, nourrissant la crainte de voir émerger un mezzogiorno allemand. Enfin, la situation financière des nouveaux Länder est désastreuse, et elle risque de s'aggraver depuis l'élargissement de l'UE et la réorientation des fonds structurels. Quel peut être dans ce contexte l'avenir des nouveaux Länder? Et comment redéfinir la politique de l'Aufbau Ost?

L'année 2004 marque un tournant dans la perception économique de la réunification. Une série de rapport d'experts pointe du doigt les sommes colossales dépensées depuis 1990 - pour de piètres résultats. Déjà, en 2003, le président de l'institut ifo, Hans-Werner Sinn, commentait ainsi les indicateurs de l'est : " ce ne sont pas des chiffres qui génèrent l'espoir d'un rattrapage». Mais le grand public n'a été alerté qu'en avril 2004 par le rapport très critique du groupe von Dohnanyi, institué par le gouvernement afin de donner un nouveau souffle à l'Aufbau Ost. Au-delà d'une analyse sans tabous de la situation, le rapport exprime surtout ce qui semblait inconcevable il y a peu encore : repenser la philosophie de l'Aufbau Ost, mettre fin aux subventions massives non ciblées et créer une "zone franche »- une Sonderwirtschaftszone Ost. Ce constat est plus ou moins partagé par d'autres rapports de toutes obédiences et origines : Conseil des Sages, DB-Research, Fondation Friedrich-Ebert, Groupe de travail sur une politique économique alternative (Arbeitsgruppe 'Alternative Wirtschaftspolitik') de l'université de Brême. Par contre, le consensus s'arrête quand s'il s'agit d'exprimer plus clairement la stratégie future : lutter, par une meilleure efficacité de la politique d'aides, contre le risque d'une 'mezzogiornisation' de l'Allemagne de l'est ou accepter la tendance globale et nuancer, voire abandonner l'objectif d'une harmonisation des conditions de vie comme le préconise le Conseil des Sages, ou encore encourager la population à déménager, comme le suggère, mi-ironique, mi-sérieux, le président de l'institut DIW ?

Ces divergences ne sont compréhensibles que par l'ampleur du marasme estallemand dont les causes remontent aux tout premiers choix faits au moment de la réunification, notamment la création, le $1^{\mathrm{er}}$ juillet 1990, de l'union économique, monétaire et sociale (UEMS). Seule réponse adéquate au plan administratif et politique (une intégration graduelle, par exemple la coexistence de deux monnaies, aurait été trop complexe à gérer, sans parler du coût psychologique pour la population et du flux migratoire vers l'ouest), I'UEMS a généré un coût
Une déferlante de rapports concluant à l'échec de l'Aufbau Ost

L'Unité aurait exigé des réformes préalables du 'modèle allemand' 
économique considérable, car elle n'était pas adaptée à la réalité économique des nouveaux Länder. Compte tenu de la faible productivité (aujourd'hui, on sait qu'elle n'atteignait que $35 \%$ de celle de l'ouest) comme des pertes d'emploi et d'actifs liées à la privatisation des ex-entreprises d'Etat, l'extension à l'est du modèle social de la RFA a présumé des forces du système social allemand. Avec ses allocations de chômage et son niveau de retraites et d'aide sociale généreux, il avait dès la fin des années 1980 commencé à toucher ses limites. Plutôt que de l'appliquer à l'est en l'état, il aurait fallu le réformer au préalable ; les réformes ne s'esquissent qu'aujourd'hui. La seconde erreur a été la mauvaise allocation du capital suite aux subventions trop massives en faveur du BTP. Elle en a d'abord alimenté artificiellement le boom, et retarde depuis 1996, début de la crise du secteur, la reprise générale. Enfin, dernière erreur : l'évolution des salaires, négociée par les partenaires sociaux, et qui, sur la base de chiffres soit inexistants, soit trop optimistes quant à la productivité, s'est soldée par un alignement beaucoup trop rapide, dommageable à l'activité et à l'emploi.

Indicateurs économiques et sociaux de l'Allemagne de l'est - 1991-2003 (Ouest=100)

\begin{tabular}{|c|c|c|c|c|c|c|c|}
\hline Indicateurs économiques & 1991 & 1997 & 2003 & Indicateurs sociaux & 1991 & 1997 & 2002 \\
\hline PIB par tête (base de prix : 1995) & 49,4 & 67,1 & 66,4 & & & & \\
\hline PIB par tête nominal & 33,4 & 62,2 & 63,9 & Revenu net par ménage & 54 & 82 & 81 \\
\hline Investissements d'équipement par tête & 62,0 & 108 & 89,0 & Salaires & 49 & 76 & 81 \\
\hline Productivité (PIB par actifs) & 51,2 & 72,5 & 74,6 & Temps de travail (par an et par tête) & 99 & 110 & 110 \\
\hline Coût salarial unitaire & 141,0 & 112,0 & 107,0 & Taux d'activité & 96 & 91 & 87 \\
\hline Stock de capitaux par tête & 38,0 & 66,0 & 71,0 & Taux de chômage & 216 & 203 & 254 \\
\hline Taux d'exportations & 52,0 & 44,0 & 60,0 & Budget social en \% du PIB généré & 184 & 156 & 164 \\
\hline Infrastructures publiques par tête & 50,0 & 64,0 & 78,0 & Budget social par tête & 61 & 95 & 100 \\
\hline
\end{tabular}

Source : Arbeitsgruppe Soziale Marktwirtschaft (2004)

La crise n'est pas typique d'un processus de transition
Les erreurs des premières années ne doivent néanmoins pas faire oublier que l'Allemagne de l'est n'a pas connu la plupart des problèmes de la transition, tels qu'on les a observés chez les voisins est-européens : privatisations retardées, crise monétaire et fiscale, crise politique, etc. Outre l'UEMS, les deux facteursclefs de ce succès sont la rapide création administrative et institutionnelle des nouveaux Länder, ainsi que le renouveau des infrastructures. L'instrument financier en a été le Pacte de solidarité Solidarpakt I, entré en vigueur en 1995 et qui, soutenu par des fonds structurels européens, a versé jusqu'à 2004 plus de 100 milliards $€$ aux nouveaux Länder. Le net retard par rapport à l'ouest en termes de capitaux par tête s'est ainsi réduit : son niveau est passé de $38 \%$ à $66 \%$ entre 1991 et 2001.

\section{L'est entre paradoxes et dilemmes}

Une productivité de $59 \%$ et un pouvoir d'achat de $90 \%$ du niveau ouest-allemand

Des retraites supérieures de $21 \%$ au niveau ouest-allemand
Tous les experts partagent aujourd'hui l'analyse selon laquelle le processus de rattrapage économique s'est arrêté depuis maintenant 8 ans. Les indicateurs économiques dressent une image cruelle. Certes, la productivité (par actif) suggère un niveau honorable : la tendance, bien que ralentie, est toujours haussière, et la productivité par actif atteint $75 \%$ du niveau de l'ouest en 2003. Mais l'indicateur économiquement plus parlant est celui de la productivité par actif occupé. II reflète l'évolution sur le front de l'emploi où le nombre de salariés est passé de 8,4 millions au début de la réunification à 7,2 millions aujourd'hui. Ainsi, la productivité n'a jamais dépassé $61 \%$. Elle est même en baisse depuis 1999 et se situe maintenant à $59 \%$, voire $56 \%$ si on ne tient pas compte de l'emploi public (près de $15 \%$ à l'est contre à peine $5 \%$ seulement à l'ouest). En même temps, les salaires sont montés en moyenne jusqu'à environ $81 \%$ du niveau de l'ouest. Certains biens et services étant moins chers à l'est de l'Elbe, le pouvoir d'achat réel atteint environ $90 \%$ du niveau occidental.

Mais ce paradoxe - faible productivité, pouvoir d'achat élevé - n'est pas le seul : le niveau des retraites est particulièrement élevé dans les nouveaux Län- 
der, grâce notamment à l'activité professionnelle généralisée des femmes du temps de la RDA. Si on ne tient pas compte des retraites des fonctionnaires ni des retraites d'entreprises (plus répandues à l'ouest), les sommes versées par les seules caisses d'assurance retraite légale par tête aux retraités est-allemands dépassent le niveau occidental de $12 \%$ en termes nominaux et de $21 \%$ en termes réels. Si on prend également en compte les programmes de préretraites (au début de la réunification, environ un demi million de personnes a ainsi mis fin à sa vie active), on voit apparaître là un des principaux leviers de cette forte augmentation des cotisations sociales outre-Rhin depuis 10 ans (plus de 5 points de pourcentage par rapport au PIB) qui sape le pouvoir d'achat des Allemands ainsi que la capacité de relance de l'économie allemande.

A l'est, le problème fondamental - depuis la réunification - est que la production propre des nouveaux Länder est toujours restée en dessous de leur consommation de biens et services. Malheureusement, la tendance s'accroît. Entre 1991 et 2002, le déficit de la balance des paiements des cinq Länder orientaux est passé de 80 milliards $€$ à 113 milliards $€$ : en 2002, pouvoirs publics, investisseurs privés et ménages ont acheté des biens et services d'une valeur de 366 milliards $€$, mais leur production n'a atteint qu'un volume de 253 milliards $€$. La différence est financée pour trois quarts par des transferts publics et pour un quart par des investissements directs et de l'endettement.

Conséquence de subventions peu ciblées, d'importantes surcapacités ont été accumulées, notamment dans le secteur du logement. A cela s'ajoute aujourd'hui que non seulement les capacités de production occidentales sont souvent suffisantes pour satisfaire la demande orientale, mais aussi que depuis la fin du boom des premières années de l'unité, les entreprises ouest-allemandes préfèrent investir à l'étranger où les marges de bénéfices sont nettement plus élevées qu'à l'est. Même si le désavantage en termes de coûts salariaux s'est réduit ces dernières années, les rendements restent handicapés à l'est par une série de facteurs : production désavantagée par des petits volumes de production et des dépenses d'énergie élevées, niveau pénalisant des charges fiscales et coûts d'approvisionnements - surtout par rapport aux voisins d'Europe de l'est. La région est littéralement prise en sandwich entre l'Allemagne de l'ouest, très productive et taillée pour être leader sur de nombreux marchés internationaux, et les nouveaux Etats membres de l'Union européenne, moins chers et plus dynamiques. L'investissement a ainsi peu d'intérêt à affluer entre l'Elbe et l'Oder, surtout quand il s'agit de créer des entreprises dans les secteurs intensifs en main d'œuvre (en atteste le recul des investissements d'équipements qui n'atteignent plus que $89 \%$ du niveau occidental, contre $108 \%$ en 1997).

Ce manque de perspectives pour l'investissement, combiné à une évolution démographique très négative - l'émigration vers l'ouest et la forte baisse des naissances rendent les conséquences du vieillissement encore plus dramatiques pour la région de l'ex-RDA que pour le reste de l'Allemagne - font craindre que l'est ne soit pris dans une spirale descendante comme on l'observe depuis des décennies pour l'Italie du Sud. Cette comparaison peut surprendre, car historiquement, les deux régions ont peu en commun. Avant la guerre, l'Allemagne de l'est représentait un noyau central de l'industrialisation allemande. La région avait profité d'une nette ascension pendant l'économie de guerre des nationalsocialistes et avait été moins touchée par des bombardements que les régions occidentales. Entre 1936 et 1944, la production y avait augmenté de plus de $50 \%$, permettant d'assurer près de $30 \%$ des biens de base allemands. Et, surtout, la productivité y était supérieure de $27 \%$ à celle de l'ouest. Le sud de l'Italie n'a jamais connu de telles évolutions. Pourtant, les deux régions présentent aujourd'hui un profil similaire : en termes de PIB par tête, le sud de l'Italie atteint à peine $60 \%$ du reste du pays (66\% dans le cas des nouveaux Länder). La productivité ( $83 \%$ contre $75 \%$ ) est relativement plus favorable du côté italien. Même chose pour la structure salariale : en Allemagne de l'est, l'emploi industriel ne représente que $15 \%$ du total contre $19 \%$ en Italie du sud.
Les nouveaux Länder consomment trop pour ce qu'ils produisent

L'est pris en sandwich entre l'ouest et les nouveaux membres de l'UE

Risque latent d'une 'mezzogiornisation'... 
... mais de grandes différences avec le sud de l'Italie

La faiblesse du Mittelstand pénalise les nouveaux Länder

Le coût brut de l'Aufbau Ost : 1500 milliards $€$ depuis 1990 !

Mais à peine $20 \%$ de cette somme est destinée à la reconstruction
Ces chiffres ne doivent néanmoins pas faire oublier une autre réalité du sud de I'Italie : l'illettrisme, une grande pauvreté et des structures maffieuses qui pénalisent son développement. Ces handicaps particuliers se traduisent par un très faible taux d'exportation : il n'atteint que $11 \%$ du niveau du reste de l'Italie (le chiffre correspondant pour les nouveaux Länder est $60 \%$ ), entretenant le sousdéveloppement de la région - une tendance que le gouvernement italien, malgré des subventions massives, surtout sur le marché du travail, n'arrive pas à inverser. Mais plus importante encore est l'absence de pôles de compétitivité dans le mezzogiorno italien. Or ces zones existent dans l'est de l'Allemagne et non pas seulement parce que la région abrite aussi la capitale Berlin. II faut rappeler dans ce contexte que la présence de régions à faible productivité est le propre de tous les grands pays industrialisés; leur absence outre-Rhin était justement ce qui a longtemps différencié l'Allemagne de ses voisins.

II n'en reste pas moins que, 15 ans après l'unification, le PIB par tête n'atteint toujours à l'est que les deux tiers du niveau occidental. Mais cette moyenne masque une forte homogénéité. Certes, la différence entre les PIB des cinq Länder est minime, mais c'est là l'effet de lissage de l'aide financière de l'ouest, notamment dans le cadre du système de péréquation entre les Länder. Une raison fondamentale de l'absence d'une dynamique autonome à l'est est la faiblesse du tissu de petites et moyennes entreprises. Seule la Saxe, aidée par sa tradition et une politique qui a misé très tôt sur ce vecteur, a réussi le maillage entre grands groupes et PME si typique du capitalisme rhénan. La Thuringe n'en est pas loin grâce au pôle d'excellence autour de Jenoptik. Mais ailleurs, les PME manquent. Ainsi, en Saxe-Anhalt, l'héritage de la prétrochimie à Leuna et de l'industrie chimique à Bitterfeld a très longtemps monopolisé toutes les énergies. II commence seulement à générer des pôles de croissance. Faute de tradition industrielle, la situation est plus délicate dans le Mecklembourg, ainsi que dans le Brandebourg, après l'échec de plusieurs grands projets.

\section{Coût élevé de l'Aufbau Ost et finances publiques en déficit}

En avril 2004, une couverture de l'hebdomadaire Der Spiegel faisait sursauter l'Allemagne : « 1250 milliards pour quoi faire ? ». Peu après, le ministre fédéral des Transports tentait de calmer les esprits en chiffrant le véritable coût de l'Aufbau Ost à 250 milliards $€$. D'où vient une telle divergence ? Le chiffre de 1250 milliards $€$ est basé sur les estimations de l'institut de Halle (IWH) pour la période de 1991 à 2003. En incluant 1990 et 2004, on obtient 1500 milliards $€$. II s'agit du total des transferts bruts, donc des sommes versées à l'est, financées par le Bund, les Länder de l'ouest et le système de protection sociale. Outre les moyens affectés aux besoins spécifiques de l'est (par exemple la reconstruction des infrastructures), ce total inclut également des dépenses classiques (comme les salaires des fonctionnaires des nouveaux Länder) et les transferts sociaux comme les allocations chômage ou les retraites.

Ce calcul ne prend pas en compte que les nouveaux Länder contribuent eux aussi à ces transferts - via les impôts et les cotisations sociales; mais leur faiblesse économique limite leur contribution à leurs propres besoins. II serait ainsi plus judicieux de considérer les transferts nets. Les nouveaux Länder contribuent à hauteur de 8 à $10 \%$ aux recettes fiscales allemandes $(10 \%$ pour la TVA, $4 \%$ pour l'impôt sur le revenu et $1 \%$ pour l'impôt sur les sociétés). Selon l'IWH, les transferts nets s'élèvent ainsi à 83 milliards $€$ en 2003, dont $45 \%$ pour le domaine social, $13 \%$ pour les infrastructures, $9 \%$ pour l'investissement, le reste pour des réalisations diverses. Pour l'ensemble de la période 1990-2004, les transferts nets se situent alors à près de 1200 milliards $€$, financés notamment par la contribution de solidarité (Solidaritätszuschlag) qui majore l'impôt sur le revenu de 5,5 points de pourcentage, l'augmentation des cotisations sociales et le gel, voire la baisse des dépenses dans les collectivités ouest-allemandes. C'est cette somme qu'il faut prendre en considération si on 
se borne à une perspective de pouvoir d'achat. Les montants alloués à la reconstruction de l'est au sens strict, qu'ils soient destinés aux travaux d'infrastructure ou aux entreprises, sont, quant à eux, nettement plus faibles. Jusqu'à la fin 2004, leur total s'élève à un peu moins de 270 milliards $€$, c'est-à-dire environ $18 \%$ des transferts bruts (pour comparaison : le budget français de 2004 s'élève à 283 milliards $€$ ). Cette relation relativise quelque peu l'actuel débat quant à l'efficacité des aides, car la large majorité des sommes qui sont allées à l'est a toujours été destinée à la consommation et non à l'investissement. Dans le même temps, ce constat a de quoi inquiéter, puisqu'il révèle aussi la faiblesse inhérente à la politique de transferts, que les instituts chargés de l'évaluation des progrès de l'Aufbau Ost critiquent en rappelant que les « effets addictifs » des subventions ont incité et incitent toujours les nouveaux Länder à vivre au-dessus de leurs moyens.

\begin{tabular}{|c|c|c|c|c|c|}
\hline \multicolumn{6}{|c|}{ Solidarpakt I et Solidarpakt II } \\
\hline \multirow{2}{*}{\multicolumn{6}{|c|}{$\begin{array}{l}\text { En 1995, le fédéralisme financier avait été élargi d'un Pacte de solidarité afin d'encadrer l'effort budgétaire supplémentaire néces- } \\
\text { saire pour intégrer les nouveaux Länder dans le système interrégional de péréquation. Il avait été conçu pour une durée de } 10 \\
\text { ans, et les Länder de l'est ont perçu } 105 \text { milliards } € \text { dans le cadre du Solidarpakt I (1995-2004). } \\
\text { Au début } 2005 \text {, le Solidarpakt II a pris le relais. Etabli pour une durée de } 15 \text { ans, il porte sur un volume de } 156 \text { milliards } € \text {. Con- } \\
\text { trairement au premier pacte, l'aide est maintenant dégressive et oblige les Länder à consolider leur situation financière. L'aide est } \\
\text { divisée entre deux pôles : le pôle } 1 \text { prévoit } 105 \text { milliards } € \text { dans le cadre des Sonderbedarfsbundesergänzungszuweisungen } \\
\text { (SoBEZ), des subventions complémentaires du Bund destinées à rattraper le retard dans les infrastructures et à soutenir les bud- } \\
\text { gets des communes à capacité financière inférieure à la moyenne (unterproportionale Finanzkraft). D'un niveau de } 10,5 \text { milliards } € \\
\text { en } 2005 \text {, son niveau baisse annuellement (surtout à partir de 2009) pour s'établir à } 2,1 \text { milliards } € \text { en } 2019 \text {, dernière année de ver- } \\
\text { sement. En contrepartie de cette dégressivité, les Länder de l'est ont obtenu que le gouvernement fédéral renonce de fait à son } \\
\text { droit de codécision quant à l'utilisation des fonds - avec comme conséquence que les Länder utilisent l'argent pour des fins autres } \\
\text { que le but d'origine. Seule la Saxe réalise une utilisation conforme au Solidarpakt II. Le pôle II porte sur un volume de } 51 \text { mil- } \\
\text { liards } € \text {. Il contient également les moyens provenant des Fonds structurels européens. Le niveau annuel n'est pas encore défini et } \\
\text { doit être négocié annuellement selon la situation financière du Bund. Pour limiter l'incertitude, les Länder de l'est souhaitent que } \\
\text { cette deuxième partie du Solidarpakt II soit inscrite dans la Loi fondamentale. }\end{array}$}} \\
\hline & & & & & \\
\hline & Saxe-Anhalt & Mecklembourg-P. & Saxe & Brandebourg & Thuringe \\
\hline \multicolumn{6}{|c|}{ Utilisation des SoBEZ pour investissements (en \%) } \\
\hline 2002 & $-9,2$ & 6,5 & 68,7 & 15,6 & 19,8 \\
\hline 2003 & $-13,8$ & 8,3 & 86,3 & 16,7 & 16,2 \\
\hline \multicolumn{6}{|c|}{ Utilisation des SoBEZ pour investissements et unterproportionale Finanzkraft (en \%) } \\
\hline 2002 & 7,9 & 23,9 & 86,3 & 34,8 & 37,4 \\
\hline 2003 & & 25,8 & & & \\
\hline
\end{tabular}

Source : Ragnitz 2004.

Malgré un montant finalement assez modeste - les transferts nets de 83 milliards $€$ en 2003 représentent à peine $4 \%$ du PIB -, le volume des aides aux nouveaux Länder est de plus en plus critiqué. Les adversaires avancent que ces transferts « épuisent » à long terme le PIB car leur niveau dépasse celui de la croissance annuelle. Cela est certes vrai. Mais la réalité est plus complexe, car ces transferts impliquent une importante redistribution en faveur de certains compartiments du secteur privé : ils sont complétés par un autre flux, celui des biens et services fournis essentiellement par les entreprises ouest-allemandes. L'est prend ainsi une place importante dans le circuit de production des entreprises ouest-allemandes. En 2000, le DIW avait estimé que les transferts exercent durablement sur le PIB ouest-allemand un effet positif de 6 à $7 \%$ par an. L'institut IW de Cologne rejoint cette analyse. II estime que le PIB ouestallemand a ainsi bénéficié entre 1990 et 2000 d'une croissance supplémentaire de 145 milliards $€$ (en termes réels). Si on prend également en compte les recettes fiscales supplémentaires générées par cet immense programme conjoncturel, ainsi que les économies réalisées par les caisses sociales du fait d'un chômage moins élevé à l'ouest (si on additionne les estimations du DIW et du IW, on arrive à plus de 90 milliards $€$ : soit plus que les 83 milliards $€$ de transferts nets), il convient bien d'admettre que les transferts ouest-est sont au moins partiellement compensés par des gains à l'ouest.
Controverse sur le mode de calcul des transferts et de leurs effets 
Aspects financiers et économiques des nouveaux Länder en 2003

\begin{tabular}{|c|c|c|c|c|c|c|c|}
\hline & Déficit $^{a}$ & $\begin{array}{l}\text { Dette totale } \\
\text { par tête }^{b}\end{array}$ & $\begin{array}{l}\text { Soutien par } \\
\text { des fonds } \\
\text { structurels }\end{array}$ & $\begin{array}{c}\text { Répartition des } \\
\text { moyens du } \\
\text { Solidarpakt II }\end{array}$ & $\begin{array}{l}\text { Crois- } \\
\text { sance }\end{array}$ & $\begin{array}{l}\text { PIB par } \\
\text { tête }^{\mathrm{g}}\end{array}$ & $\begin{array}{l}\text { Popu- } \\
\text { lation }\end{array}$ \\
\hline Mecklembourg-P. & 3,32 & 6675 & 2,44 & 10,5 & 0,8 & 17,1 & 1,74 \\
\hline Berlin & 5,78 & - & ${ }^{d} 0,73$ & 19,0 & 0,4 & 20,9 & ${ }^{d} 1,25$ \\
\hline Brandebourg & 2,22 & 7010 & 2,98 & 14,3 & 0,9 & 17,5 & 2,58 \\
\hline Saxe-Anhalt & 3,1 & 7902 & 3,23 & 15,7 & 0,9 & 17,4 & 2,55 \\
\hline Thuringe & 2,92 & 6726 & 2,78 & 14,3 & 1,7 & 17,6 & 2,39 \\
\hline Saxe & 0,71 & 3917 & 4,69 & 26,1 & 2,3 & 17,8 & 4,35 \\
\hline Ensemble de la RFA & 3,7 & nd & - & - & 1,7 & 27,4 & 81,3 \\
\hline
\end{tabular}

Sources : Gouvernement fédéral, ministère des Finances, Destatis, Commission européenne. (a) en \% du PIB du Land ; (b) Land et communes, en millions $€$; (c) en milliards $€$; (d) Berlin-Est ; (e) en \% ; (f) en 2004, en \% par rapport à 2003 ; (g) en milliers (h) en millions.

Endettement par tête élevé à l'est... mais aussi à l'ouest

Des aides financières assurées jusqu'en 2019
Cette compensation ne réduit en rien le caractère dramatique de la situation est-allemande. En termes absolus, la dette cumulée de l'ensemble des collectivités des nouveaux Länder a atteint 85 milliards $€$ en 2003 - soit à peu près la somme perçue la même année au titre des transferts nets. L'endettement par tête (en intégrant la dette des communes) dans les nouveaux Länder est monté à $6274 €$ à la fin 2003. Si on y ajoute l'endettement du Bund (9 $345 €$ ), l'endettement total de chaque Allemand de l'est s'élève à plus de $15600 €$. Si ce niveau reste en dessous du niveau occidental (plus de $16000 €$ en 2003), il doit être supporté par un PIB par tête qui n'atteint qu'à peine deux tiers du niveau de l'ouest. Les causes de cette situation se trouvent des deux côtés du budget. La capacité à autofinancer les dépenses est restée très basse à l'est : en 2003, la part des recettes fiscales propres permettant de couvrir les dépenses n'atteint que $34 \%$. Mais l'est dépense également trop. Si on prend les quatre Länder les plus pauvres (à l'est : le Brandebourg, le Mecklembourg, la Saxe-Anhalt et la Thuringe ; à l'ouest : la Basse-saxe, la Rhénanie-Palatinat, la Sarre et le Schleswig-Holstein), on constate qu'à l'est les moyens financiers sont de $20 \%$ plus élevés, tandis que le déficit par tête (toutes collectivités confondues) est plus important à l'est qu'à l'ouest ( $574 €$ contre $514 €$, chiffres pour 2003). Seule exception : la Saxe, avec $142 €$ de déficit par tête en 2003. La situation des finances publiques est indéniablement très mauvaise à l'est, mais elle l'est également dans beaucoup de Länder de l'ouest, et l'actuel débat quant aux déficits publics et la contrainte de Maastricht se trompe de cible en prenant l'est comme seul bouc émissaire.

Au vu de ces chiffres, il n'est guère étonnant que les nouveaux Länder utilisent de plus en plus souvent les aides du Solidarpakt I pour combler des trous budgétaires - pour assurer tout simplement les dépenses courantes comme les salaires des fonctionnaires. A plusieurs reprises déjà, le ministre fédéral des Finances a exprimé son fort mécontentement quant à cette utilisation peu conforme des aides à l'investissement; mais les termes du pacte le condamnent à l'impuissance. Les tensions risquent de s'intensifier avec le Solidarpakt II qui vient d'entrer en vigueur et qui prévoit le versement de 156 milliards $€$ aux cinq Länder de l'est sur la période 2005-2019. Dans ce pacte non plus, ces flux n'ont pas d'affectation fixe. Autre pomme de discorde : la remise en question de certaines aides de l'UE. Actuellement, les nouveaux Länder perçoivent environ 20 milliards $€$ par an au titre des fonds structurels européens (FSE). A partir de 2007, ce montant devrait être revu à la baisse du fait de l'élargissement de l'UE. Mais la stagnation persistante à l'est pourrait en limiter la réduction, car les critères de soutien pour la période 2007-2013 seront établis sur la base des années 2001 à 2003. Et le dernier rapport de cohésion de la Commission européenne montre qu'en 2001, l'ensemble du territoire est-allemand à l'exception de Berlin, Dresde, Leipzig et de certaines parties du Brandebourg, se situait audessous du seuil de $75 \%$ de la moyenne du PIB de l'UE justifiant les subventions les plus élevées (Objectif 1). En 2000, seules les régions de Dessau et de Chemnitz étaient concernées par l'Objectif 1 (rattrapage des régions en retard 
de développement). Mais aujourd'hui, en période de renégociation des FSE, les Länder est-allemands sont amenés à dramatiser quelque peu la réalité de leur retard pour prolonger les subventions...

\section{Pourquoi l'Aufbau Ost a-t-il échoué et comment faire mieux?}

En Allemagne, c'est la déception qui domine désormais quant à l'Aufbau Ost, et on noircit dès lors volontiers le tableau. Pourtant, il existe des secteurs et des pôles au dynamisme indéniable. Dans certains villes et régions (Leipzig, Chemnitz, Rostock, Potsdam... pour n'en citer que quelques-unes), la situation est non seulement très satisfaisante, elle est également meilleure que dans certains régions de la Sarre, de la Basse-Saxe ou même de Bavière. Néanmoins, il faut admettre que dans sa globalité, la réalité est-allemande est encore très loin de ces "paysages fleuris » promis par Helmut Kohl en 1989. La liste des explications à cet échec relatif est longue et connue : taux de conversion trop généreux, privatisations hasardeuses, salaires disproportionnés, non-affectation des transferts, etc. De même que celle des 'coupables' présumés: responsables politiques, entreprises ouest-allemandes trop peu «patriotiques »... Mais aurait-on pu faire mieux ? Difficilement, étant donné l'urgence et l'ampleur du chantier. Et par-delà les griefs de circonstance, on peut distinguer trois raisons principales permettant d'expliquer la situation actuelle : 1) I'héritage historique et la nature même de la transition;2) l'évolution salariale ; 3) la dérive des pratiques de cohésion territoriale liées à l'ordre constitutionnel de la RFA.

Aujourd'hui, le débat sur le niveau et l'évaluation du soutien financier à l'est a tendance à oublier un peu hâtivement certaines données historiques ainsi que les caractéristiques inévitables du processus de transition. Après 1945, les régions est-allemandes ont subi leur première désindustrialisation massive sous le régime soviétique, se manifestant par le démontage de l'industrie et des infrastructures, évalué à un tiers de la capacité industrielle de 1944. S'y ajoutent les réparations financières, estimées à environ 13 milliards de $\$$ (aux prix de l'époque). Le niveau des réparations par tête était ainsi quatre fois plus élevé pour les citoyens de l'ex RDA que pour ceux de la RFA. Après 1990, l'est a subi une deuxième désindustrialisation suite à la restructuration et à la dissolution des anciennes entreprises d'Etat dans le cadre des opérations de la Treuhandanstalt. D'autres facteurs s'y sont ajoutés, dont la perte des marchés en Europe de l'Est. Au total, la production industrielle est-allemande a ainsi baissé de près de deux tiers entre 1989 et 1992. Même en 1997, elle n'atteignait qu'à peine $50 \%$ du niveau d'avant l'Unité. Malgré les spécificités est-allemandes, cette évolution est typique et probablement inévitable: en effet, le PIB de presque tous les pays en transition a traversé une courbe en ' $U$ ' avec un fort effondrement après la libéralisation économique, suivi d'un rattrapage progressif.

Le second facteur expliquant l'échec de la réunification économique est le niveau des salaires et le rattrapage trop rapide. Souvent, cette tendance est justifiée par le taux de conversion très favorable de l'Ostmark en DM. Cependant, malgré un taux de près de la parité, les salaires orientaux n'atteignaient qu'environ un tiers du niveau occidental au début des années 1990. Le gros du rattrapage s'est joué après. C'est d'autant plus regrettable, car avec des salaires à un tiers du niveau de l'ouest, l'est aurait facilement concurrencé toute l'UE de l'époque et attiré massivement des investissements étrangers - avec pour conséquence que les salaires auraient remonté tout naturellement, mais avec une productivité qui aurait eu le temps de suivre. La réalité a malheureusement été une autre, car presque tout le mouvement de rattrapage s'est réalisé dans la première moitié des années 1990. II a ainsi devancé sensiblement l'évolution de la productivité. La responsabilité de cette évolution est partagée entre les acteurs politiques et les partenaires sociaux: le patronat ouest-allemand pour avoir cherché à contenir la " concurrence déloyale » des entreprises est-allemandes, les syndicats pour avoir cherché à enrayer le risque d'un « dumping
3 raisons : histoire, salaires, dérive du principe de cohésion territoriale

Deux désindustrialisations depuis 1945

L'erreur cardinale d'un rattrapage salarial trop rapide 
La dérive des pratiques liées à l'objectif de cohésion territoriale

Une politique régionale inadaptée, car basée sur le saupoudrage

Des conditions de vie « équivalentes » et non homogènes social », et le pouvoir politique pour avoir préparé le terrain en se mêlant massivement dans les négociations conformément au slogan « à travail égal, salaire égal ».

Plus encore que les deux premiers facteurs, c'est la dérive (antérieure à l'Unité) des pratiques liées à l'ordre constitutionnel de la RFA qui explique l'échec relatif de la politique de l'Aufbau Ost. Celui-ci repose sur les principes organisant la cohésion territoriale. Le premier est celui des «tâches communes » (Gemeinschaftsaufgaben) qui permet de déroger dans certains cas à la répartition des compétences législatives entre le Bund et les Länder au nom de l'intérêt conjoint. L'art. 91 a $\S 1$ de la Loi fondamentale stipule ainsi que le Bund « concourt à l'accomplissement des tâches des Länder dans les secteurs suivants, si ces tâches sont importantes pour l'ensemble et si ce concours ... est nécessaire à l'amélioration des conditions de vie ". Ce concours est d'ordre financier: le Bund contribue à hauteur de $50 \%$ aux dépenses nécessaires. Les secteurs concernés sont au nombre de trois, dont « l'amélioration de la structure économique régionale " (Verbesserung der regionalen Wirtschaftsstruktur). Voilà qui légitime les aides à l'investissement pour les entreprises et les collectivités. L'autre principe-clef est celui de la solidarité territoriale à la base du système de péréquation financière dans le cadre du fédéralisme financier (Länderfinanzausgleich), appliqué dans le Solidarpakt I et II. Or il apparaît aujourd'hui que ni l'un ni l'autre de ces principes ne se sont révélés adaptés à la reconstruction de la structure économique est-allemande, car leur mise en œuvre avait subi au fil des ans une dérive favorisant une pratique de saupoudrage indifférencié des aides plutôt qu'une politique de soutien ciblé. Et ils se sont traduits par une série d'effets pervers qui inhibent aujourd'hui la dynamique de croissance des nouveaux Länder (et de certains Länder ouest-allemands) au lieu de la libérer.

Les Gemeinschaftsaufgaben, inscrites dans la Loi fondamentale à la fin des années 1960, ont toujours été une politique qui visait des territoires très limités, ainsi que l'harmonisation des conditions de vie entre des territoires où les disparités étaient somme toute assez faibles. Lors de la réunification, on a appliqué cette politique à une région avec des problèmes d'une toute autre nature. L'ex-RDA a des structures qui sont beaucoup moins favorables au succès de cette politique car la région dispose de peu de zones à forte concentration. Malgré ce fait, l'Aufbau Ost se base jusqu'à ce jour sur l'attribution uniforme, sans considération des différences régionales, de volumes financiers redistribués dans le cadre du fédéralisme financier. Outre le fait que les Gemeinschaftsaufgaben contreviennent à l'organisation du fédéralisme qui, dans le partage des compétences, confie l'autonomie administrative aux Länder (cette contradiction est débattue dans la réforme en cours du fédéralisme), ces «tâches communes », de même que le Länderfinanzausgleich, ont non seulement généré une attitude revendicatrice de la part des Länder qui en bénéficient, mais les incitent également, en socialisant le financement de certaines tâches, à un comportement d'autant plus dépensier qu'aucun Pacte de stabilité interne à la RFA ne les contraint à respecter une certaine discipline budgétaire. L'actuel souhait des nouveaux Länder de faire inscrire le Solidarpakt /I dans la Loi Fondamentale en est une illustration.

La politique de subventionnement massif est également le fruit de la dérive d'un autre principe constitutionnel encore : la "réalisation de conditions de vie équivalentes » (art. 72 § 2) qui légitime l'intervention législative du Bund au nom de l'intérêt supérieur de "l'ensemble de l'Etat » dans le cadre des compétences législatives concurrentes qui confèrent aux Länder "le pouvoir de légiférer aussi longtemps et pour autant que la Fédération n'a pas fait par une loi usage de sa compétence législative » (art. $72 \S 1$ ). Cet article, aujourd'hui sur la sellette dans le cadre plus général de la réforme du fédéralisme puisqu'il a contribué à une centralisation rampante des compétences, s'appliquait nécessairement lors de l'unification au nom de la solidarité nationale. Ce dernier principe confère en outre au Bund le droit de rééquilibrer les capacités financières entre les Länder 
en leur accordant des dotations complémentaires afin de « les aider à couvrir leurs besoins financiers généraux » (art. 107 § 2, al. 3) - mais sans qu'il soit question de conditions de vie «équivalentes ». Or c'est l'interprétation à accorder à cette dernière notion qui fait aujourd'hui débat. A l'origine, le qualificatif figurant dans la Constitution était : « homogènes » (Einheitlichkeit). Or au fil du temps, l'interprétation de ce concept avait abouti à une dérive égalitariste vers l'idée d'uniformité des conditions de vie. L'article 107 de la Loi fondamentale avait dès lors été modifié en 1994, et le qualificatif " homogènes » remplacé par "équivalentes » afin de rendre plus transparent l'esprit de ce principe, à savoir l'équité des chances. Et dans plusieurs arrêts rendus depuis, le Tribunal constitutionnel fédéral a rappelé qu'il implique l'acceptation de certaines disparités et ne peut donc se confondre avec une approche égalitaire, considérée comme contraire aux principes fondateurs de l'ordre constitutionnel libéral et démocratique de la RFA. Pour mieux expliciter ce retour à l'orthodoxie, la Cour avait par exemple formulé le principe de «l'interdiction du nivellement » (Verbot der Nivellierung) dans son arrêt du 11-11-1999 sur le Länderfinanzausgleich.

Au niveau fédéral, la remise en cause de cette politique de soutien indifférencié vient tout juste de commencer. Avec une croissance qui depuis 1996 reste audessous du niveau de l'ouest (entre 1996 et 2001, le PIB a progressé de 1,5\% par an contre 1,7\% à l'ouest), la région n'aura pas les moyens de se hisser au même niveau que l'ouest. Car à long terme, ce n'est pas la demande (largement subventionnée), mais l'offre qui suscitera la croissance. Cette offre est déterminée par le travail et le capital disponibles, ainsi que le progrès technique. Même si on suppose que le stock de capital s'accroît à l'est (il ralentit depuis 1995), l'évolution démographique condamne la région : sa population diminue et vieillit plus rapidement que celle de l'ouest. Le rattrapage qui ne s'est pas réalisé jusqu'ici pourra difficilement se faire à l'avenir. Mais ceci ne condamne pas la région dans son entier : comme à l'ouest, la différenciation territoriale s'accroîtra à l'est ; seules quelques zones atteindront le niveau de l'ouest.

Aujourd'hui, l'Allemagne cherche à donner un nouveau souffle à l'Aufbau Ost. Si la proposition de constituer une Sonderwirtschaftszone Ost (une sorte de zone franche) revient régulièrement dans le débat (mais sa réalisation est peu compatible avec la réglementation européenne), la tendance est plutôt à la concentration des aides dans des clusters, c'est-à-dire des zones à grappes d'activités spécialisées et ouvertes à la concurrence internationale. La Saxe et la Thuringe ont montré le chemin. Les chiffres sont parlants : en 2004, la Saxe a enregistré avec la Bavière la plus forte croissance (2,3\%) économique des Länder allemands. II n'est guère étonnant dès lors que la large majorité des rapports d'experts préconise maintenant la concentration des aides dans des clusters, d'autant que cette approche a fait par ailleurs ses preuves en Bavière ou en Rhénanie du Nord-Westphalie, et qu'elle semble bientôt porter ses fruits dans la région Berlin-Brandebourg (voir REA 40/99, 62/03 et 68/04). A l'est, les gouvernements ont déjà commencé une réorientation de la politique en ce sens. Ainsi, le Mecklenbourg et le Brandebourg sont en train de se positionner dans le secteur des services et de l'industrie agro-alimentaire. Par ailleurs, le Brandebourg profite de la proximité de Berlin qui fait que plusieurs grappes industrielles se sont déjà implantées autour de la capitale, mais sur le territoire brandebourgeois. Et même le Mecklembourg se met à exploiter son potentiel indéniable d'énergie éolienne.

L'ACtUELle deCEPTION CONCERNANT L'Aufbau OST est nourrie par deux facteurs: d'un côté l'optimisme démesuré quant à la rapidité du rattrapage qui avait prévalu au cours des premières années de l'unité allemande ; de l'autre, une confiance trop aveugle dans le système politique, économique et en particulier social, qui a voulu ignorer trop longtemps la nécessité de réformes structurelles. De ce point de vue, l'échec de l'Aufbau Ost est aujourd'hui le point de cristallisation des deux principaux maux allemands: un système social trop
Quelques zones seulement réussiront le rattrapage

Vers la concentration sur les zones à fort potentiel de croissance 
généreux compte tenu d'une capacité économique stagnante et d'une société vieillissante, d'une part, et un système fédéral dont l'approche liant concurrence et solidarité à la fois s'est dénaturée au fil du temps, aboutissant à une centralisation rampante et à un système de péréquation financière qui incite trop à l'assistanat et pas suffisamment au développement de la compétitivité économique. Ces problèmes sont identifiés, et de timides reformes sont engagées. Quant à l'est - et finalement à l'Allemagne tout entière - un autre phénomène s'y ajoute. Le "modèle allemand " ne pourra plus à l'avenir garantir le même niveau 'd'égalité' que celui auquel les Allemands se sont habitués pendant si longtemps. Face à des difficultés budgétaires et mue par son cap de réformes (stratégie de Lisbonne), l'Allemagne rompt avec la dérive égalitaire, source de nivellement. Finalement, c'est un processus de normalisation qui traverse aujourd'hui l'Allemagne - une normalisation structurelle impliquant l'acceptation des disparités qui existent également à l'ouest et dont la croyance en ces fameux «paysages fleuris » avait masqué un peu la perception.

A l'est, seules certaines zones ont déjà réussi leur rattrapage ou y parviendront, d'autres resteront durablement à la traîne. Cette évolution est inévitable compte tenu du développement démographique. Le scénario développé pour l'est en 2020 par le Berlin-Institut paraît dans ce contexte très réaliste : "En 2020, on pourra se promener entre la mer Baltique et le Fichtelgebirge sans rencontrer beaucoup de personnes... Avec le vieillissement, la nature reprendra ses droits. Elle enclenchera une dynamique écologique sans précédant dans l'histoire moderne de l'Allemagne. La diminution de la population et sa concentration dans des régions à forte plus-value modifiera la conception de l'espace. D'importantes sommes financières deviendront disponibles et pourront être réorientées vers des utilisations plus productives, comme l'enseignement et la recherche". Mais ce scénario s'appliquera aussi à de larges territoires du Schleswig-Holstein, de la Basse-Saxe ou même de la Bavière...

\section{Indications bibliographiques :}

AG 'Alternative WiRTSChaftSPolitiK', "Gegen die Zwangsperspektive des ostdeutschen Zurückbleibens - für forcierte Mobilisierung endogener Entwicklungspotentiale Ost », Universität Bremen, 2004

BACH S., VeSPER D., « Finanzpolitik und Wiedervereinigung. Bilanz nach 10 Jahren », DIWVierteljahresheft, $n^{\circ} 2 / 2000$

BerLIN-INSTITUT Für WeLtBeVÖLKERUNG UND GLOBALE ENTWICKLUNG, Deutschland 2020, Berlin, 2004

BOURGEOIS I., " 10 ans après l'unification: l'économie des nouveaux Länder en voie de normalisation", Regards sur l'économie allemande, n46/00, et "Quelle politique pour les nouveaux Länder dans l'UE 25 ? ", Regards sur l'économie allemande, $\mathrm{n}^{\circ} 61 / 03$ BUNDESREGIERUNG, Jahresbericht 2004 zum Stand der deutschen Einheit, 2004 Busch U., Am Tropf. Die Ostdeutsche Transfergesellschaft, Berlin 2002

DB RESEARCH, « Perspektiven Ostdeutschlands, 15 Jahre danach », n`306, 2004

DIW / IAB / IfW / IWH / ZEW, Fortschritte beim Aufbau Ost. Fortschrittsbericht wirtschaftswissenschaftlicher Institute über die wirtschaftliche Entwicklung in Ostdeutschland, 2002

DRÜKE H., «Europas Stiefel drückt und zwickt - Grundprobleme der Wirtschaft Italiens », Aus Politik und Zeitgeschichte, B 35-36, p. 23, 2004

FES-MANAGERKREIS, Doch noch ein Wirtschaftswunder in Ostdeutschland ?, mai 2004

GABEL M., "La réforme du fédéralisme financier allemand: beaucoup de bruit pour rien? ", Regards sur l'économie allemande, $n^{\circ} 52-53 / 01$

LALLEMENT R., "L'économie est-allemande : transition inachevée et réorientation de la politique économique ", Note du Cerfa, n¹7, novembre 2004

LAMmERS K., "Problemregion Ostdeutschland - was ist zu tun? », IW-Wirtschaftsdienst, $n^{\circ} 10 / 2004$

RAGNITZ J., «Zur Ausgestaltung des Solidarpaktes II“, IWH Diskussionspapier, n¹94/2004

RAGNITZ J., " Transferleistungen für die neuen Länder », Wirtschaft im Wandel, n`9-10/2004

SACHVERSTÄNDIGENRAT, Jahresgutachten 2002-2003 et 2004-2005

SinN H.-W., Ist Deutschland noch zu retten?, Econ, 2003 